\title{
Full-Reference and No-reference Image Blur Assessment Based on Edge Information
}

\author{
David B.L. Bong, Adeline S.L. Ng \\ Faculty of Engineering, Universiti Malaysia Sarawak, 94300 Kota Samarahan, Malaysia. \\ E-mail:bbldavid@feng.unimas.my
}

\begin{abstract}
Blur images are often subjected to the loss of high frequency content during acquisition, compression and multimedia transmission. Hence, objective blur assessment is implemented to identify and quantify image quality degradation by blurriness artifact in order to maintain and control the quality of the images. In this paper, objective full-reference and no-reference blur assessments using edge information are presented with the aim to provide computational models that can automatically measure the amount of blurriness artifact such as Gaussian blur on the images. The amount of Gaussian blur on an image, also known as the final blur measurement is determined by averaging the sum of edge width over all detected edges which satisfy the edge criteria. The final blur measurement for all test images based on full-reference and no-reference implementations are also validated with subjective results. The validation results show that the objective full-reference and no-reference blur assessments correlate closely to perceptual image quality.
\end{abstract}

Keywords — Edge; full-reference; Gaussian blur; no-reference .

\section{INTRODUCTION}

Along with the rapid advances in digital and multimedia imaging industry, digital images have been playing an increasingly important role in the communication of visual information. Unfortunately, these images can be degraded during acquisition, compression, transmission or even processing. Thus, a measure to the image quality is necessary to assess the degree of degradation. The method of measuring the degree of degradation is categorized into objective and subjective evaluations. Objective method is based on mathematical measure while subjective method relies on the perception of a selected group of human observers such as professionals or lay viewers. Objective image assessment is least preferable as the most reliable means to access the image quality is through subjective evaluation, where the quality of the image is evaluated by human. However, it is not an easy task to conduct a subjective evaluation image assessment because it is expensive and time-consuming [1]. Due to the limitations in subjective evaluation, researchers believed that it is useful to design objective method as long as it produces results that correlate closely with human visual system (HVS) [2]. There are 3 approaches to objective image quality assessment that can be considered such as noreference (NR) measurement, reduced-reference (RR) measurement and full-reference (FR) measurement [2]. A FR measurement requires complete availability of the original image while NR method accesses the distorted image without using any reference image. Meanwhile, RR method needs partial information of the reference image and it is a trade-off between both NR and FR methods.

Researchers have developed various perceptual image quality metrics where these metrics are used to measure the global distortion but a perceptual objective image quality analysis to measure a specific artifact such as blur is rarely found. Therefore, this paper aims to propose an objective blur assessment using edge information which correlates well with human visual perception based on FR and NR implementations.

\section{BLUR ASSESSMENT}

Most digital imaging capture devices and electronically displaying visual information devices aim to produce the best image quality. When an artifact like blur is introduced in an image due to acquisition or compression, the blur image has to be enhanced in order to look visually appealing. However, there is a possibility that this corrected image might not satisfy human perception. Various people evaluate the quality differently due to the sensitivity of human eye. Therefore, the blur metric which correlates with human visual as according to P. Marziliano et al. [3] is 
introduced to measure the blur image to determine the level of degradation so that it can be corrected to a certain extent to produce a better quality image and maintain the pleasure of human observers in viewing the image.

There also other researches in blur assessment such as a No-reference Quality Metric for Measuring Image Blur presented by E. Ong et al. [4] in 2003 using the gradients' directions of each pixel in the image, The Blur Effect: Perception and Estimation with a New No-Reference Perceptual Blur Metric proposed by F. Crete et al. [5] in 2007 by comparing the variations between neighboring pixels while X. Wang et al. [6] published a Blind Image Quality Assessment for Measuring Image Blur in 2008 which is also based on edge gradient information. Although in [4] and [5], the researchers mentioned that their metrics correlate well with results obtained from subjective experiments but the correlation values are not reported. Moreover, in [6], the authors did not mention about validating their metric with subjective scores. However, the blur assessment using edge information presented in this paper which is based on the method implemented by $\mathrm{P}$. Marziliano et al. [3] is highly correlated with subjective blur ratings when measuring on Gaussian blurred images. Based on Pearson linear correlation and Spearman's rank order reported in [3], the metric has shown $96 \%$ correlation with its subjective evaluation.

Nevertheless, the blur measurement is applicable to numerous applications such as blur estimation in digital photography, image processing, printing or as a simple metric in comparing two images.

\section{METHODOLOGY}

\section{A. Test Images}

There are two sets of images used as test images. The first set consists of synthetically generated blur artifact on 29 RGB original images from LIVE database [7] using Gaussian blur lowpass filter of kernel size $15 \times 15$. Each of the images is blurred using Gaussian standard deviations, $\sigma$ $\in\{0.4,0.8,1.2,1.6,2.0\}$. This set of images is used for blur assessment implementation in Section IV-A until Section IV-C.

In the second set, these test images are used for analysis in Section IV-D. There are 29 original images blurred using circular-symmetric 2-D Gaussian kernel of standard deviations ranging from 0.42 to 15 pixels by $\mathrm{H}$. Sheikh et al. [8] to generate 145 blur images. Each source images has five blur images distorted with different standard deviation.

The total test image in each set is 174 images including original and distorted images. Fig. 1 shows some of the test images used for the blur assessment.

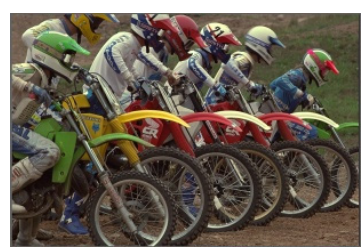

(a)

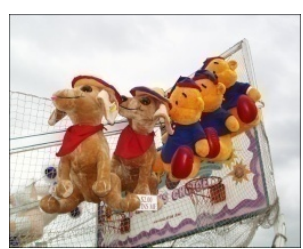

(b)

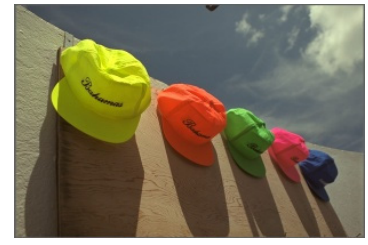

(c)

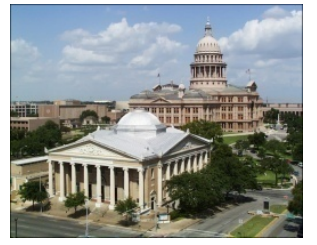

(d)
Fig. 1 Original test images: (a) bikes (b) carnival dolls (c) caps (d) church and capitol

\section{B. NR Implementation}

The blur image is converted to $\mathrm{YCbCr}$ color space as blurriness is measured on luminance $(\mathrm{Y})$ component. The vertical Sobel edge detector is applied to the Y component image to determine the vertical edges. Blurriness can be measured on gray-level component by converting the RGB test image to grayscale image. The processing steps are illustrated in Fig. 2 below.

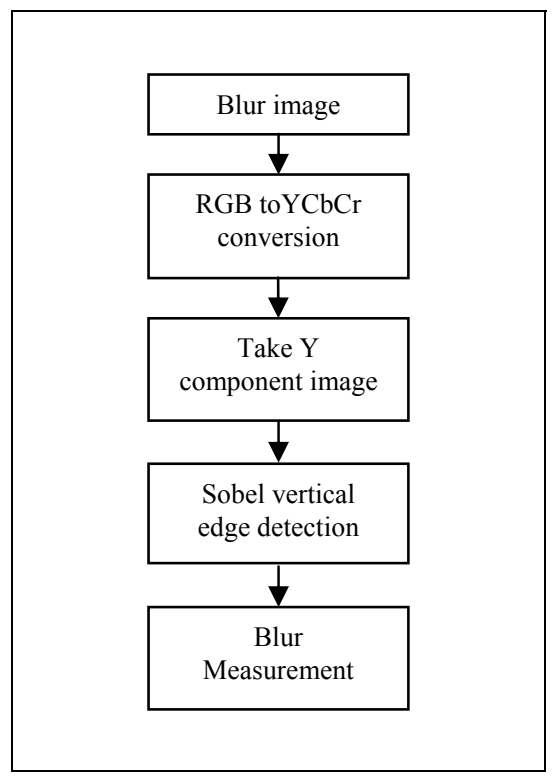

Fig. 2 Flow chart of NR implementation

\section{FR Implementation}

The original image is required for this implementation. Both original and blur images have to perform $\mathrm{YCbCr}$ color conversion. The vertical Sobel edge detector is then applied on the $\mathrm{Y}$ component image from the original image to obtain the location of the edges. As shown in Fig. 3, the blur measurement is based on the Y component of the blur image and edge locations detected from the original image. 


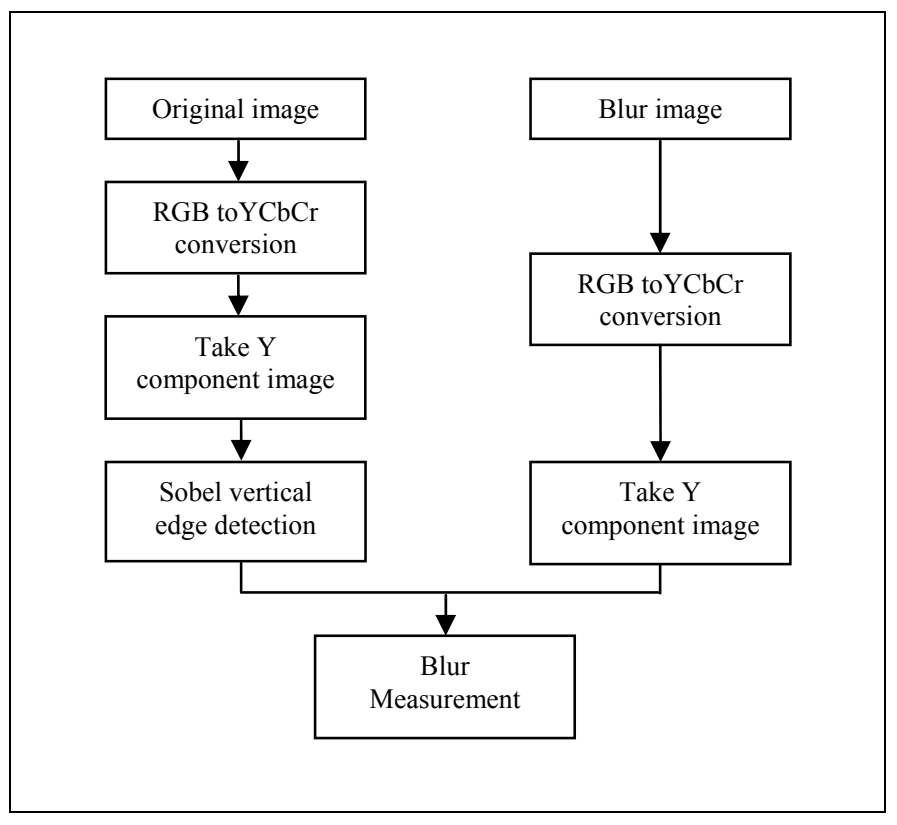

Fig. 3 Flow chart of FR implementation

\section{Blur Measurement}

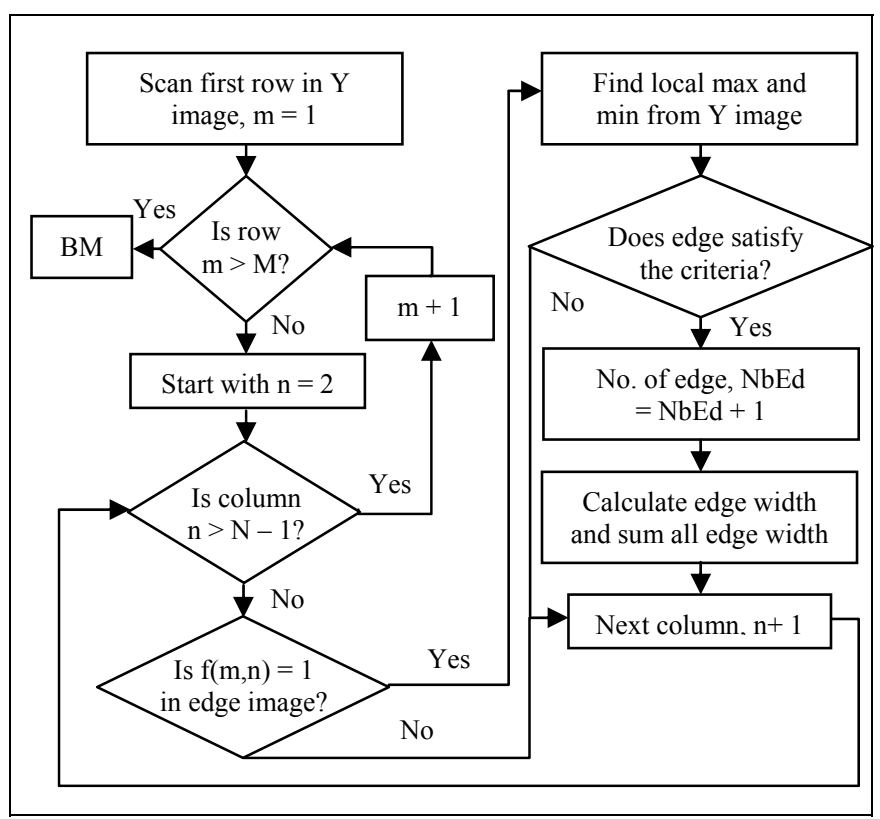

Fig. 4 Flow chart of blur measurement algorithm for an image having $M$ rows and $N$ columns

The blur measurement algorithm is summarized in Fig. 4. First, scan the first row of the $Y$ component image. For each edge detected from edge detection, the local minimum and maximum positions are determined. These positions are known as the start and end positions of the edge. They are also defined as the local extrema locations closest to the edge.

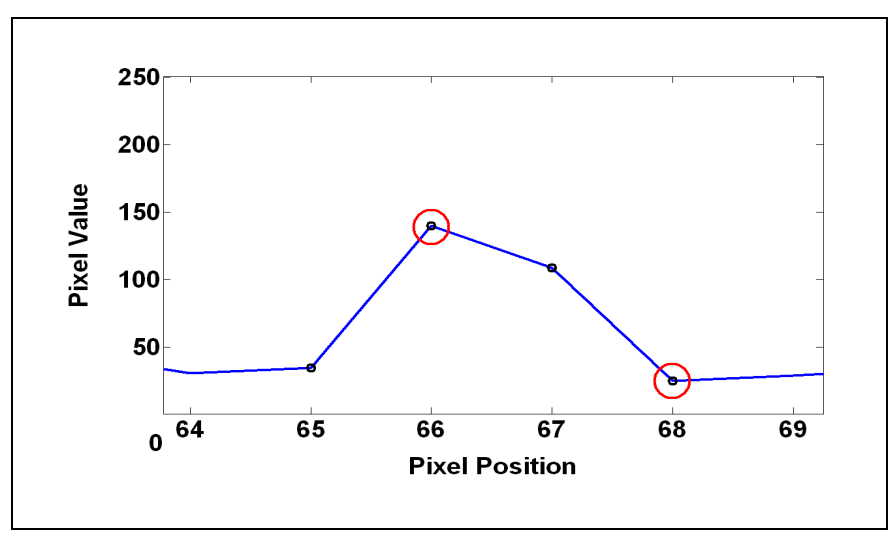

Fig 5 Edge falls on local maximum and local minimum positions

After determining the start and end positions, the edge must satisfy the edge criteria because experimentally, insignificant edges are detected from Sobel edge detection. For example, insignificant edges which are highlighted by the red circle on the line, fall on the same position as the start (local maximum) and end (local minimum) positions as illustrated in Fig. 5 above. These edges are not supposed to fall on such positions because it is not possible to obtain the edge width for these edges. Other types of insignificant edges are such that the edges fall on the start or end pixel position in some particular row in the image.

Thus, the edge criteria are introduced to overcome this problem. The edge is only accepted based on certain conditions as follows:

- An edge must reside in between a local minimum and a local maximum.

- An edge cannot fall on the start or end pixel position in every row of an image.

- An edge cannot fall on the same pixel position as the local minimum or local maximum.

Hence, once the detected edge satisfies the criteria, the edge width of the accepted edge is calculated. The edge width is given by the difference between the start and end positions. It is also known as the local blur measure for an edge location. The procedure of calculating the edge width and number of edges is repeated for every row in the image. The total blur measurement of a blur image is summarized as,

$$
\text { Blur metric }(\mathrm{BM})=\frac{\sum \text { Edge width }}{\text { Total number of edges }}
$$

An example is shown in Fig. 6 below on calculating the edge width for an edge which falls on position P1. The edge width for this edge is given by the difference between P2 (start position) and P2' (end position), which is 7 pixels for this example. 


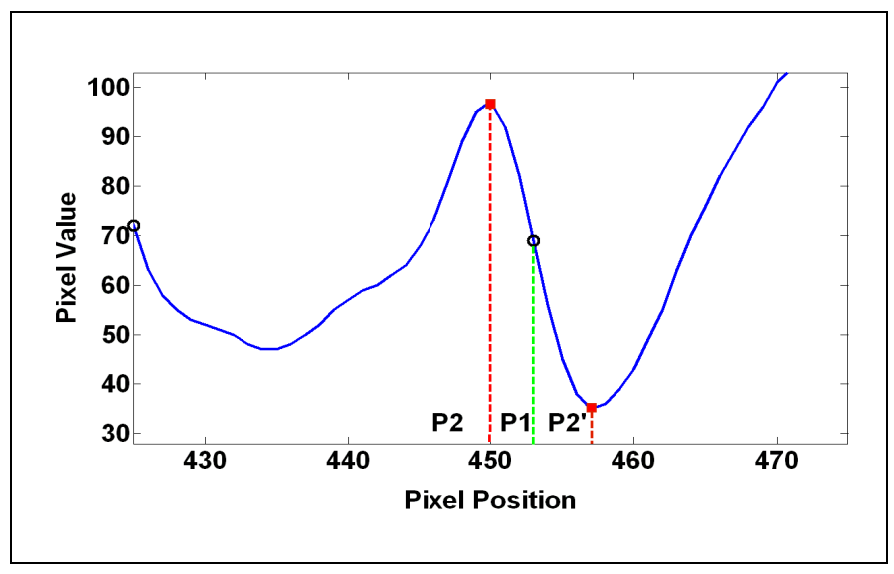

Fig. 6 Calculating edge width for an edge at position P1

\section{RESUlTS AND DiSCUSSION}

This section summarizes and discussed the results from implementation and results from each analysis.

\section{A. NR Blur Assessment on Luminance Component}

It is clearly seen from the graph in Fig. 7 that the metric increases with the standard deviation. This is true as blurriness artifact increases (standard deviation value increases), the image becomes smoother. Thus, the edges become thicker resulting in the increase of edge widths. As image becomes smoother, the number of detected edges decreases. From equation (1), the increase of sum of edge width and the decrease of total number of detected edges eventually increase the blur measurement. The results for this graph are also validated with the results by $P$. Marziliano et al. [3] on the same image which is illustrated in Fig. 8. Both graphs appear to be similar. Based on the subjective evaluation conducted by P. Marziliano et al. [3], there is a high correlation rate of $96 \%$ between the blur measurement and human perception. This shows that the measurement follows well with subjective interpretation.

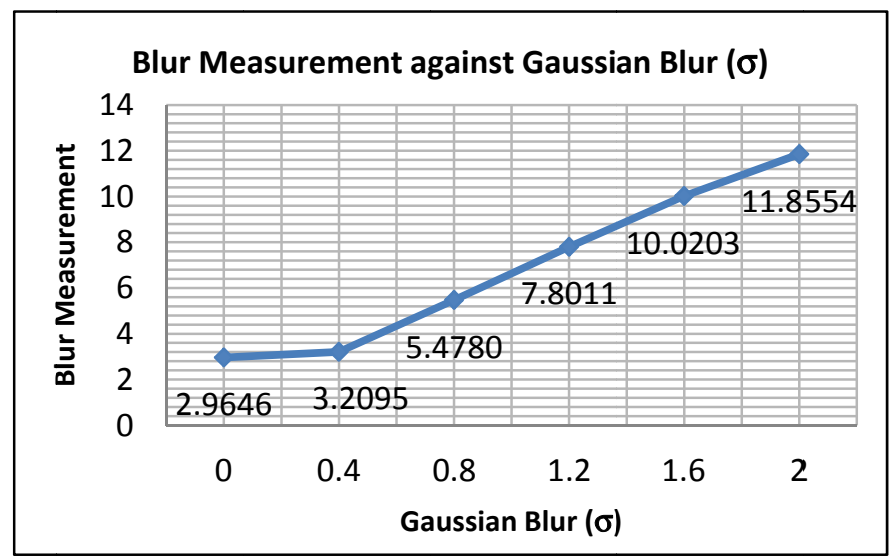

Fig. 7 NR blur assessment results for bikes test image

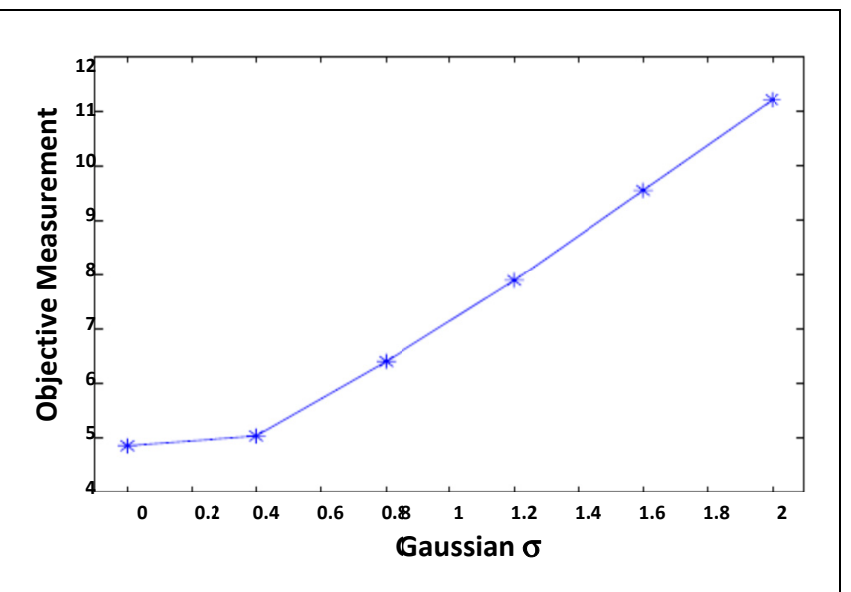

Fig. 8 NR blur assessment results for bikes test image provided by $\mathrm{P}$. Marziliano et al. [3]

\section{B. FR Implementation}

Based on the comparison graph in Fig. 9, the FR implementation tends to provide lower metric values than that of NR implementation.

The variation of the measurements is expected because in NR implementation, the edge detector is applied on the distorted image while in FR implementation, edges are detected in the original image. Thus, using NR method, as blur increases, it decreases the number of edges detected by Sobel filter. Also, the edge width for each detected edge is larger when the level of blurring is higher. However, for FR implementation, the increase of the degree of blurriness does not correspond to the number of edge detected.

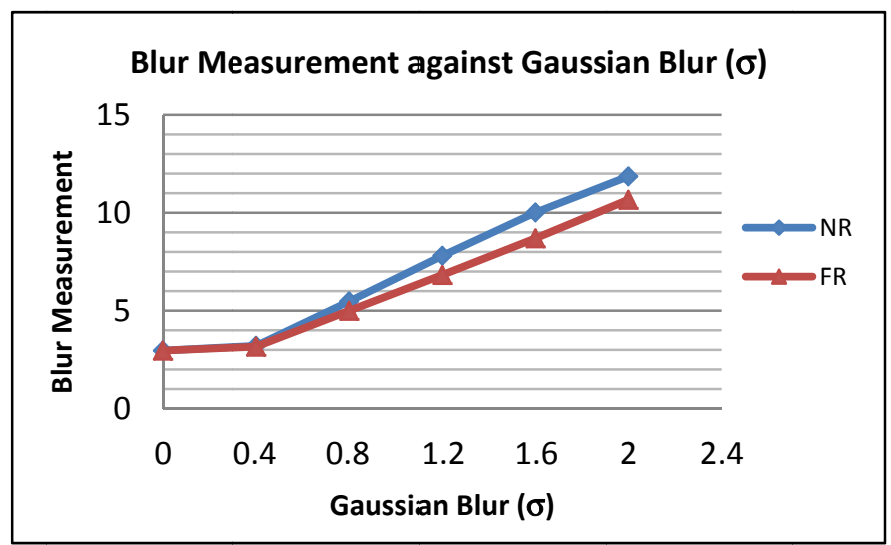

Fig. 9 Comparison between NR and FR blur assessment

\section{Analysis on NR Blur Assessment}

There are three types of analysis done on NR blur assessment such as follows:

1) on grayscale componemt: This analysis is compared with the results of NR blur assessment on Y component. The graphical result in Fig. 10 shows that the difference in blur measurement between two component images is negligible. The graph of blur measured on luminance component closely follows the same pattern of the graph of blur measured on grayscale component. This applies to most of the test images. 


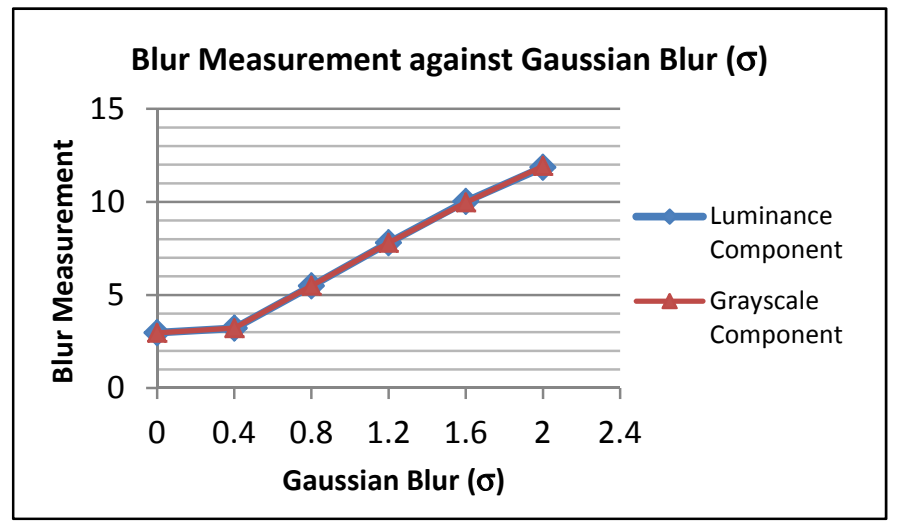

Fig. 10 Comparison between NR blur assessment on luminance component and grayscale component for bikes test image

2) using grayscale images: This analysis is to compare with the blur measurement in Section IV-C-1. Graphically, the results are identical as seen in Fig. 11.

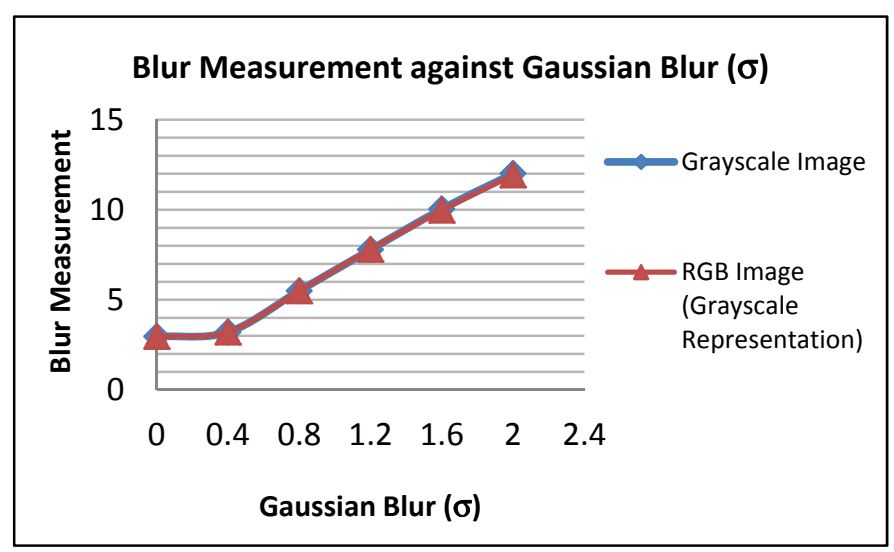

Fig. 11 Comparison between NR blur assessment using grayscale image and RGB image represented by grayscale component of bikes test image

3) horizontal edge information: The NR blur measurement presented by P. Marziliano et al. [3] considers only vertical edges due to performance reasons. However, the blur assessment can be easily extended to horizontal edge detection. Fig. 12 shows that blur metric based on horizontal edge detection is higher than the metric value from vertical edge detection. This applies to most of the test images.

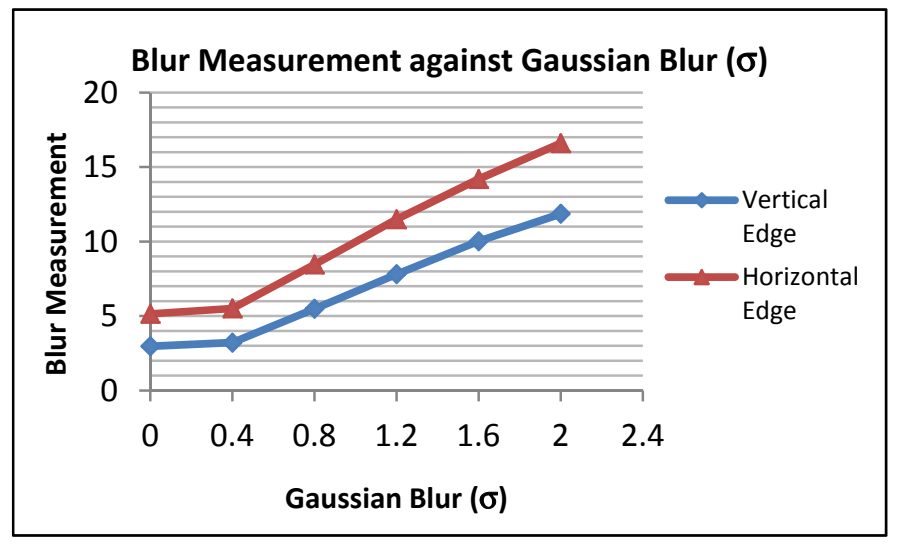

Fig. 12 Comparison between NR blur assessment based on vertical edge detection and horizontal edge detection

\section{Analysis using LIVE Database Gaussian Blur Images}

Below are the blur assessment implementations using blur images from LIVE database [7]. The result of each implementation is validated with subjective testing result (DMOS values) from LIVE database [7].

1) NR Implementation: The scatter plot in Fig. 13 shows a relatively high correlation between the objective and subjective results with $\mathrm{R}^{2}$ equivalent to 0.81 . $\mathrm{R}^{2}$ value close to 1 indicates excellent linear reliability and correlation.

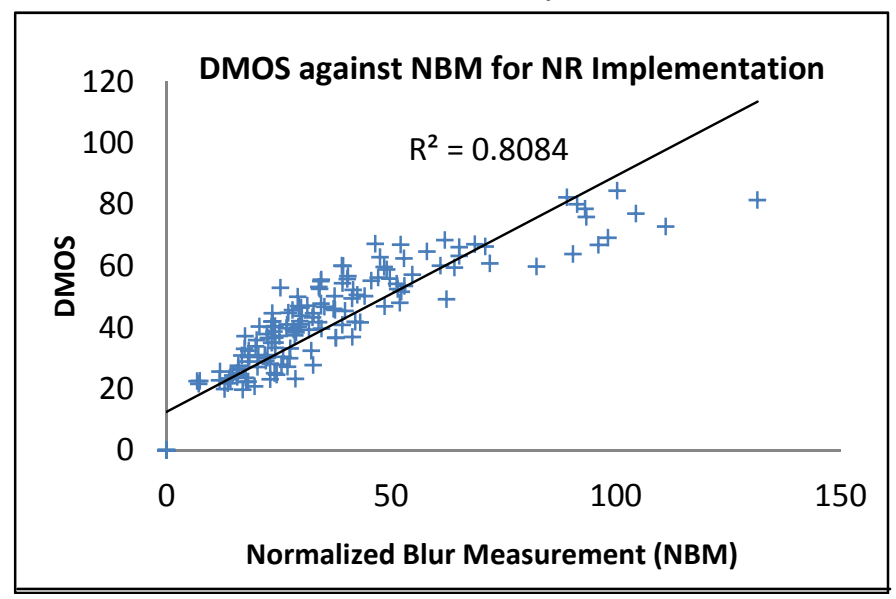

Fig. 13 Correlation between NR normalized blur measurement and DMOS values

2) FR Implementation: Fig. 14 illustrates the blur measurement for all test images with respect to the subjective results. The blur metric performs well and the value of $\mathrm{R}^{2}$ is 0.87 indicates high correlation.

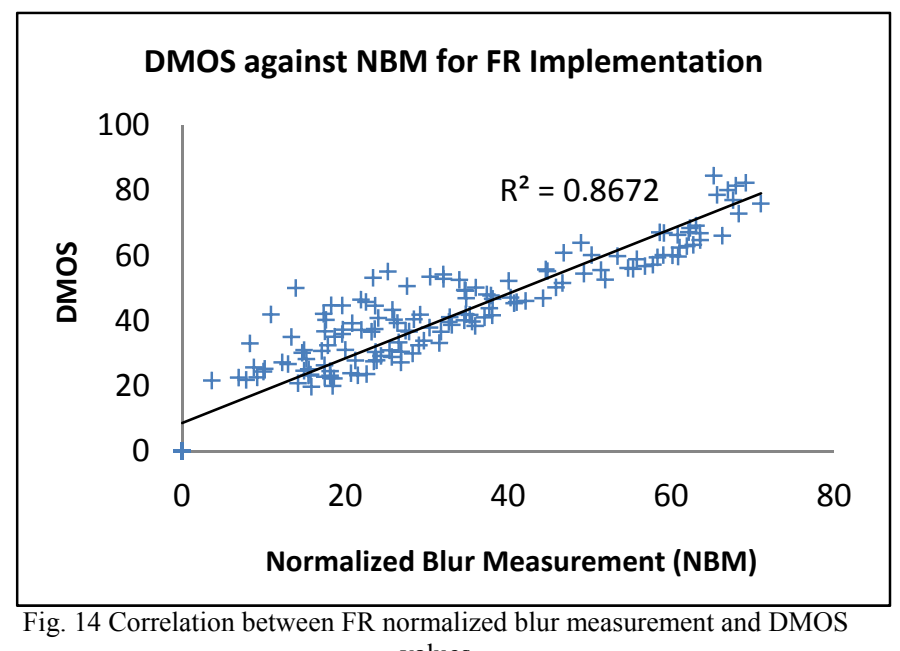

values

3) Line fitting method (to obtain normalized blur measurement): This method is to align the linear graph of the objective results closer to the nonlinear graph of the subjective results. First, the difference between the blur measurement of the original image and blurred image is obtained. Then, the square root of the difference in measurement (SDBM) is calculated and represented as,

$$
\mathrm{SDBM}=\sqrt{\mathrm{BM}_{\mathrm{BLUR}}-\mathrm{BM}_{\mathrm{ORI}}}
$$


where, $\mathrm{BM}_{\mathrm{BLUR}}$ denotes the raw blur measurement of blur images and $\mathrm{BM}_{\mathrm{ORI}}$ is the raw blur measurement of original image.

The graph of SDBM against DMOS values is then plotted for every blur level in the image and both lines are aligned using Microsoft Excel trendline function. A gain, $\alpha$ is obtained from the average of DMOS and SDBM line gradients. Thus, the gain is multiplied with SDBM values to acquire the normalized blur measurement (NBM) values.

\section{E. Discussion}

Based from the results, the blur metric increases with Gaussian blur standard deviation. The increase of degree of blurriness in an image produces a smoother image, hence this affects the number of detected edges to decrease and the edge width of each detected edge to increase. Thus, from equation (1), the blur metric eventually increases due to the increase of sum of edge width and the decrease of total number of edges.

Moreover, the metric values based on FR implementation are lower than that of the metric values from NR implementation. This is because in FR method, the increase in blur level does not affect the number of detected edges.

In both Section IV-C-1 and Section IV-C-2, the results indicate that the comparison graphs are similar. Meanwhile, Section IV-C-3, it is observed that the horizontal edge detection does not improve the measurements. For the analysis in Section III-D, the results from NR and FR blur assessments correlate closely to subjective results.

Concisely, the blur assessment has low computational complexity. The total computational time for measuring blur on all 174 test images in any method of implementation (NR or FR) is approximately 160 seconds. Hence, the elapsed time of measuring blur artifacts on an image is less than 1 second.

\section{CONCLUSIONS}

A low computational complexity objective blur assessment is successfully implemented to measure the degree of blurriness in an image. Different types of analysis are also carried out to evaluate the performance of the blur assessment. The results of the assessments are as well validated with subjective results where the validation indicates that there is a close correlation between the objective blur assessments with human perception.
As for further work, the blur assessment can be easily extended to digital video quality assessment to measure the level of blur in every consecutive frame in the video. The global blur measurement of a video will be the average of local blur measurement in every successive frame. Hence, the blur assessment can be improved so that is also applicable to real-time application. Besides that, blur identification algorithm is recommended to implement together with the blur assessment. Instead of measuring the amount of blur artifacts that present in an image, the type of blur that degrades the image can be identified so that user will have knowledge on which type of blur distorts the image quality. Finally, further research can be done on the blur assessment by measuring blurriness in color images on other color component such as hue instead of measuring on the luminance component.

\section{ACKNOWLEDGMENT}

The authors would like to acknowledge Faculty of Engineering, Universiti Malaysia Sarawak for the provision of research facilities.

\section{REFERENCES}

[1] Z. Wang, G. Wu, H. R. Sheikh, E. P. Simoncelli, E. -H. Yang, and A. C. Bovik, "Quality-aware images," IEEE Transactions on Image Processing, vol. 15, no. 6, pp. 1680 - 1689, June 2006.

[2] Z. Wang, H. R. Sheikh, and A. C. Bovik, "Objective Video Quality Assessment," in The Handbook of Video Databases: Design and Applications, B Furht and O. Marqueseds, Eds. Boca Raton, FL: CRC, 2003, pp. $1041-1078$.

[3] P. Marziliano, F. Dufaux, S. Winkler, and T. Ebrahimi, "A noreference perceptual blur metric," in Proc. of the International Conference on Image Processing, Rochester, NY, 2002, pp. 57 - 60.

[4] E. Ong, W. Lin, Z. Lu, X. Yang, S. Yao, F. Pan, L. Jiarrg, and F. Moscheni, "No-reference quality metric for measuring image blur," in Proc. of IEEE International Conference on Image Processing, Sept. 2003, pp. $469-472$.

[5] F. Crete, T. Dolmiere, P. Ladref, and M. Nicolas, "The blur effect: perception and estimation with a new no-reference perceptual blur metric," in Proc. of SPIE on Human Vision and Electronic Imaging XII, San Jose, California, USA, Jan. 2007.

[6] X. Wang, B. Tian, C. Liang, and D. Shi, "Blind image quality assessment for measuring image blur," in Proc. of Congress on Image and Signal Processing, 2008.

[7] H. R. Sheikh, Z. Wang, L. Cormack and A.C. Bovik, "LIVE Image Quality Assessment Database Release 2", http://live.ece.utexas.edu/res earch/quality.

[8] H. R. Sheikh, M. F. Sabir and A. C. Bovik, "A statistical evaluation of recent full reference image quality assessment algorithms", IEEE Transactions on Image Processing, vol. 15, no. 11, pp. 3440 - 3451, Nov. 2006 